\title{
Effect of particle size on nonlinear refractive index of Au nanoparticle in PVA solution
}

\author{
Esmaeil Shahriari, W.Mahmood Mat Yunus, ${ }^{*}$ and Elias Saion \\ Department of Physics, Universiti Putra Malaysia, 43400UPM, Serdang
}

(Received on 24 March, 2010)

\begin{abstract}
Nonlinear refractive index of Au nanoparticle suspended in PVA solution was measured using a single beam Z-scan technique. Measurements were carried out using a green $\mathrm{CW}$ laser beam operated at $532 \mathrm{~nm}$ as excitation source. Five nanoparticle samples with different particle sizes were prepared by $\gamma$ radiation method. The $\mathrm{Au}$ nano-fluid shows a good third order nonlinear response for particle sizes ranging from $7.0 \mathrm{~nm}$ to $18.7 \mathrm{~nm}$. The sign of the nonlinear refractive index was found to be negative and the magnitude was in the order of $10^{-8}$ $\mathrm{cm}^{2} / \mathrm{W}$. The results show that the nonlinear effect tends to be increased linearly with the increasing of particle sizes thus could be a good candidate for nonlinear optical devices.
\end{abstract}

Keywords: Nonlinear properties, Z-scan technique, nanoparticles.

\section{INTRODUCTION}

Nonlinear optical materials have increasing interest over the past twenty years, due to the numerous applications in various fields such as telecommunications, optical data storage and information processing [1]. Metal nanoparticles have been studied extensively because of their large third-order nonlinear susceptibilities and nonlinear optical response [2,3] and attractive for many applications such as electronic and optical devices [4], chemical and biological sensors [5-8], optical energy transport [9-12] and thermal therapy [13].

A single beam Z-scan technique developed by the Mansoor Sheik-Bahae et al. in 1990 [14] is a simple and effective tool for determining the nonlinear susceptibility. It has been used widely in material characterization because it provides not only the magnitudes of the real and imaginary parts of the nonlinear susceptibility, but also the sign of the real part [1518]. This method utilizes a tightly focused laser beam that is intense enough to access nonlinearities in a sample. As the sample passes through the focal point of the beam, changes in its transmittance due to nonlinear absorption (NLA) and nonlinear refraction (NLR) are measured using an open aperture and closed aperture experimental set up, respectively. In the open aperture technique, after the beam passed through the sample, it is focused directly into a detector. As the sample travels through the focus of the initial beam, the transmittance either increases or decreases (depending on the nonlinearity of the sample) and the detector receives more or less light than the linear transmittance, yielding a hump or dip in the curve of transmittance as a function sample position. For NLR, after the beam passing through the sample, it is attenuated by a semi-closed aperture that usually allows less than $30 \%$ of the initial beam to be detected by the detector. The converging and diverging of the beam (allowing more and less of the beam to pass through the aperture, respectively) due to the changes in the refractive index, a pre-focal valley and post-focal peak are observed for a positive change in refraction. While a pre-focal peak and a post-focal valley is observed for a negative change in refraction.

The effect of concentration on nonlinear refraction coef-

*Electronic address: mahmoodescience. upm. edu.my ficient of Au nano-fluid in PVP has been measured and reported in our previous work [19]. Since increasing the $\gamma$ radiation dose decreases the particle size of metal nanofluid, it is of our interest to study the effect of particle size on the nonlinear refractive index of such material. Therefore in this paper we report the effect of particle size on nonlinear refraction coefficient of Au-PVA nanofluid measured using a single beam Z-scan technique.

\section{EXPERIMENTAL}

In the present work, hydrogen tetrachloroaurate (III) hydrate, premion (metals basis), ( $\mathrm{HAuCl}_{4} .3 \mathrm{H}_{2} \mathrm{O}$ with $99.999 \%$ purity), 2.5 g polyvinyl alcohol, PVA (MW 29,000 Aldrish), and $1 \mathrm{ml}$ isopropanol were used for preparing Au nano-fluid sample. The PVA and isopropanol were used as a colloidal stabilizer and hydroxyl radical scavenger, respectively. The PVA solution was made by dissolving PVA powder in $50 \mathrm{ml}$ deionized water at room temperature. The solution was magnetically stirred for $2 \mathrm{~h}$ and was bubbled with nitrogen gas (99.5\%) to remove oxygen. Hydrogen tetrachloroaurate (III) hydrate, $\mathrm{HAuCl}_{4} \cdot 3 \mathrm{H}_{2} \mathrm{O}$ at weight of $2 \mathrm{mg}$ was added into PVA solution to produce Au nano-fluid sample at a concentration of $1.471 \times 10^{-4} \mathrm{M}$ and was subsequently irradiated at different doses of $\gamma$-radiation. The $\gamma$-radiation (60Co-rays) was used as an effective tool for polymerization process and reducing agent. The radiation dose was varied from 10 to $70 \mathrm{kGy}$ to obtain sample with various nanoparticle sizes as listed in Table 1. In this process, $\gamma$-irradiation produces hydrated electrons that reduced the gold ions to gold atoms, which then aggregated to certain size in the solution depending on the exposure time of radiation. The average diameters of Au nanoparticles were measured using Nanophox machine (Sympatec GmbH, D-38678) and the TEM measurements were carried out to confirm the particle size, shape and uniformity of the specimens. The average particles sizes of the samples are listed in Table 1.

The schematic diagram of a single beam Z-scan experiment used in the present measurement is shown in Figure 1 as clearly illustrated in our previous report [19]. The experiments were performed using a $532 \mathrm{~nm}$ laser beam from Laser-diode (Coherent Compass SDL-532-150T). The beam was focused to a small spot using a lens and the sample was moved across the focal region along the $\mathrm{z}$-axis by a motorized translational stage. The beam waist was $24.4 \mu \mathrm{m}$. The 
TABLE I: Nonlinear optical properties of Au nano-fluid measured at $532 \mathrm{~nm}$ laser beam

\begin{tabular}{ccccc}
\hline $\begin{array}{c}\text { (Au-PVA) } \\
\text { samples }\end{array}$ & $\begin{array}{c}\text { Average Particle } \\
\text { size }(\mathrm{nm})\end{array}$ & $\mathrm{n}_{2}\left(\mathrm{~cm}^{2} / \mathrm{W}\right)$ & $\alpha_{\left(\mathrm{cm}^{-1}\right)}$ & $\mathrm{D}\left(10^{-4} \mathrm{~cm}^{2} / \mathrm{s}\right)$ \\
\hline S1 & 7.0 & $-3.14 \times 10^{-8}$ & 2.102 & 19.9 \\
S2 & 9.9 & $-3.20 \times 10^{-8}$ & 2.220 & 20.8 \\
S3 & 10.5 & $-3.25 \times 10^{-8}$ & 2.373 & - \\
S4 & 13.0 & $-3.36 \times 10^{-8}$ & 2.420 & 22.32 \\
S5 & 18.7 & $-3.50 \times 10^{-8}$ & 2.733 & 24.49 \\
& & & & \\
\hline
\end{tabular}

transmitted light in the far field passed through the aperture and was recorded by a detector. All the measurements were carried out at room temperature for both closed aperture and open aperture configurations using a $2 \mathrm{~mm}$ sample cell. The linear absorption spectra and thermal diffusivity values of the present samples were measured using UV-Vis spectrophotometer (Shimadzu-UV1650PC) and a double beam thermal lens method, respectively.

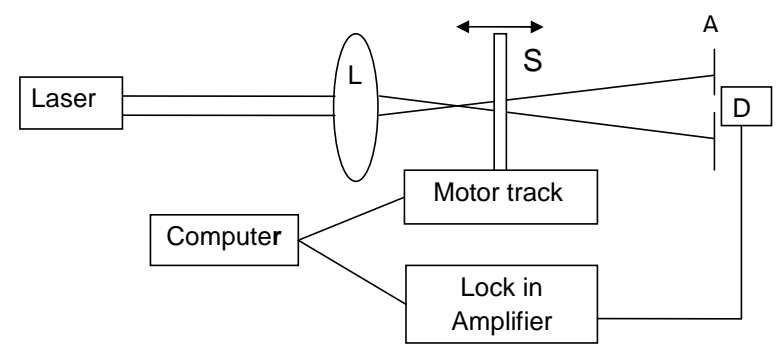

FIG. 1: Schematic diagram of Z-scan experimental setup: L- Lens, S- Sample, A- Aperture, D-Detector.

\section{Results and discussion}

Figure 2 shows the absorption spectra of the Au nano-fluid prepared using $\gamma$-radiation. The measurements of absorption spectra were carried out at room temperature for visible wavelength ranging from $360 \mathrm{~nm}$ to $800 \mathrm{~nm}$. The spectra show that the surface plasmon absorption peak appeared at 524 is not affected by the particle size increasing from $7.0 \mathrm{~nm}$ to $18.7 \mathrm{~nm}$. The particle size can be controlled by controlling the doses of $\gamma$-radiation, however in the present work the specimens were prepared at a concentration of $1.471 \times 10^{-4}$ $\mathrm{M}$ and irradiated at different doses to produce various particles sizes. Figure 3 displays a typical TEM image showing the particle with a uniform distribution and uniform shape. The average particle size obtained from TEM images was $18.7 \mathrm{~nm}$ which agrees very well with the particle size measured using Nanophox machine.

Figures 4-8 show the closed aperture Z-scan curves obtained for Au nano-fluid at same concentration but different in particle sizes. The squared symbols represent the experimental data while the solid lines are theoretical fits to the

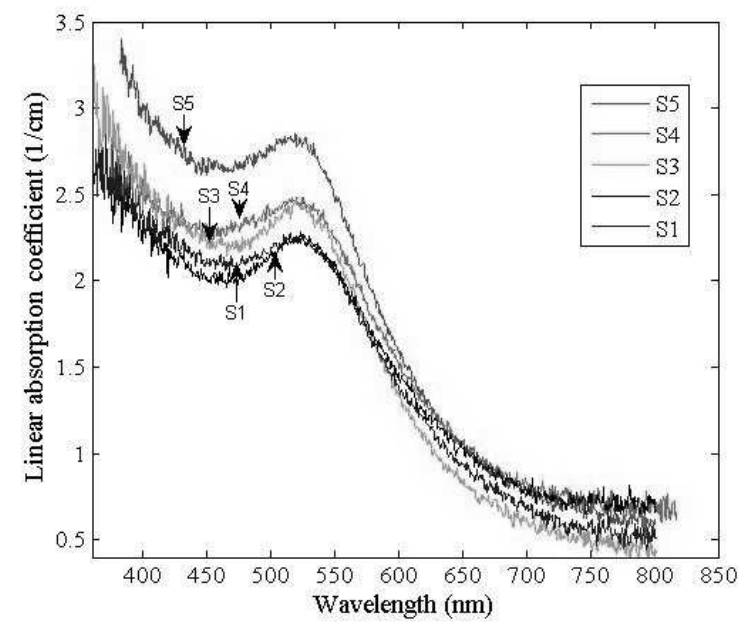

FIG. 2: Absorption spectra of Au nano-particle solution at concentration of $1.471 \times 10^{-4} \mathrm{M}$ and different sizes; S1- $7.0 \mathrm{~nm}$; S2- 9.9 nm; S3- $10.5 \mathrm{~nm}$; S4- $13 \mathrm{~nm}$; S5- $18.7 \mathrm{~nm}$.

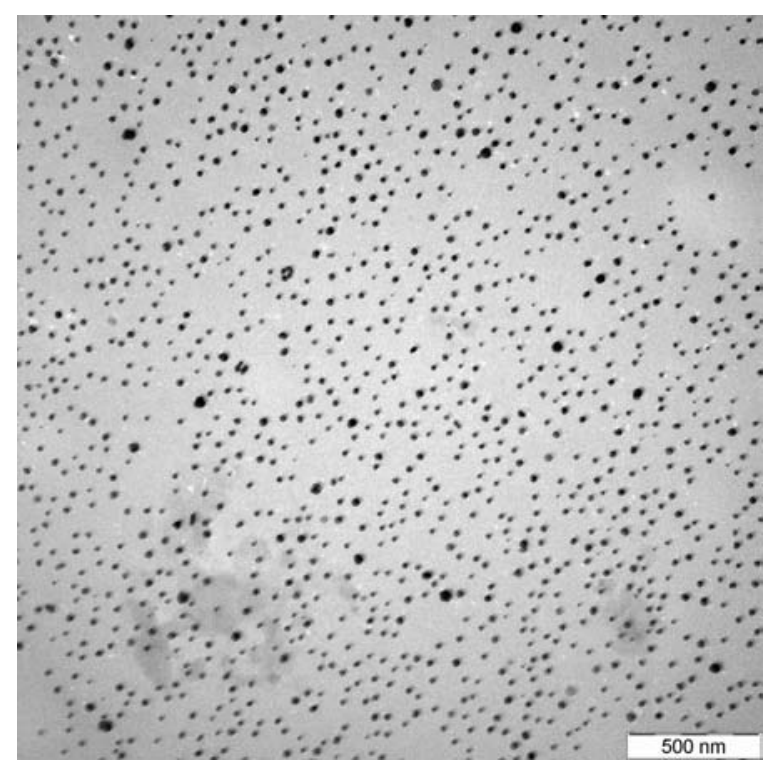

FIG. 3: TEM image of Au particles with a nominal size of $18.7 \mathrm{~nm}$.

closed aperture using standard equations given as [14]

$$
T(z, \Delta \phi)=1+\frac{4 \Delta \phi_{o} x}{\left(x^{2}+1\right)\left(x^{2}+9\right)}
$$

where $x=z / z_{0}, z_{0}$ is the Rayleigh length (3.52 $\left.\mathrm{mm}\right), \Delta \phi_{0}=$ $k n_{2} I_{0} L_{e f f}$, is the phase change due to the nonlinear refraction, $n_{2}$ is the nonlinear refractive index, $k=2 \pi / \lambda$ is the wave vector, $I_{0}=4.27 \times 10^{3} \mathrm{~W} / \mathrm{cm}^{2}$ is the on-axis irradiance at focus (i.e., $\mathrm{z}=0$ ), and $L_{e f f}=\left[1-\exp \left(-\alpha_{0} L\right)\right] / \alpha_{0}$ is the effective length of nonlinear medium, $\alpha_{0}$ is the linear absorption coefficient of the samples and $\mathrm{L}$ denotes the sample thickness $(2 \mathrm{~mm})$. The theoretical transmittance curves presented in Figures 4-8 fit very well to the experimental data and show perfect symmetry curves indicating nonlinearity 
absorption coefficient is very small. This was confirmed that all the open aperture Z-scan curves for the present sample are linear (not shown).

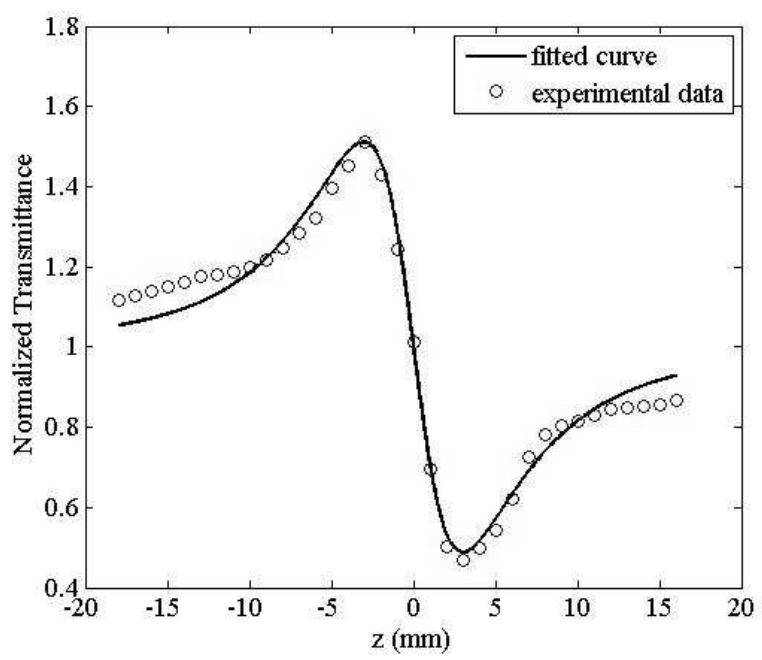

FIG. 4: Closed aperture Z-scan experimental curve for (Au-PVA) nano-fluid measured for sample with particle size of $7.0 \mathrm{~nm}$.

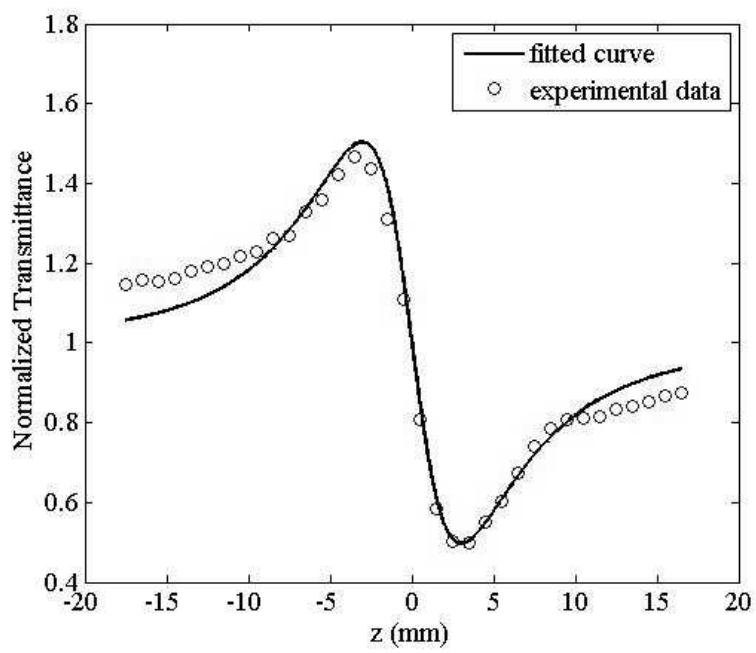

FIG. 5: Closed aperture Z-scan experimental curve for (Au-PVA) nano-fluid measured for sample with particle size of $9.9 \mathrm{~nm}$.

The nonlinear refractive index, $n_{2}$ was calculated from $\left(\Delta T_{P \rightarrow V}\right)$, where the value of peak to valley of data transmittance from the closed aperture Z- scan measurement can be described as [14]

$$
\Delta T_{p-v} \approx 0.406(1-s)^{0.25}\left|\Delta \phi_{o}\right|
$$

Here $s$ is the linear transmittance of the aperture. The nonlinear refraction coefficients $n_{2}\left(\mathrm{~cm}^{2} / \mathrm{W}\right)$ together with the values of linear absorption and thermal diffusivity of all samples obtained in the present work are listed in Table 1 and clearly indicates the self-defocusing phenomenon. In Figure 9 we

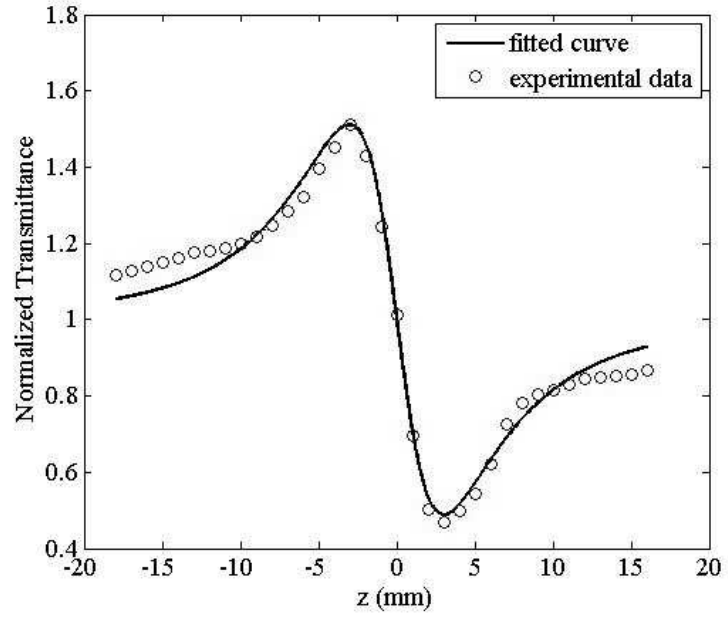

FIG. 6: Closed aperture Z-scan experimental curve for (Au-PVA) nano-fluid measured for sample with particle size of $10.5 \mathrm{~nm}$.

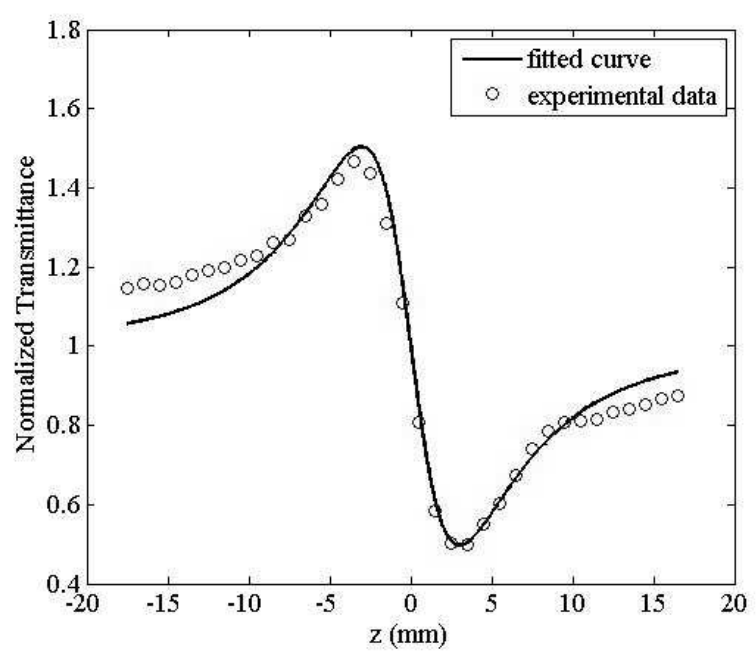

FIG. 7: Closed aperture Z-scan experimental curve for (Au-PVA) nano-fluid measured for sample with particle size of $13.0 \mathrm{~nm}$.

show the variation of the nonlinear refraction index coefficient as a function of particle sizes. We observed the nonlinear refraction coefficient tends to increase linearly with the increasing of particle sizes. This nonlinear refractive index particle size dependence is totally different with the one recently reported in the literature [19]. Thus the nonlinear refraction behaviour of Au-PVA nanofluid was affected by the sample synthesizing technique.

Since the effective thermal nonlinearity of the medium can be written as [20]

$$
n_{2}^{t h}=\left(\frac{d n}{d T}\right) \frac{\omega_{0}^{2}}{4}\left(\frac{\alpha}{k}\right)
$$

where $k=D \rho C_{p}, D$ is thermal diffusivity of the nanofluid. For small changes of $\mathrm{dn} / \mathrm{dT}$, the nonlinear refractive index solely depends on the ratio of $\alpha / \mathrm{D}$. In the present work the value of $\alpha / \mathrm{D}$ increases with the increasing of particle size 


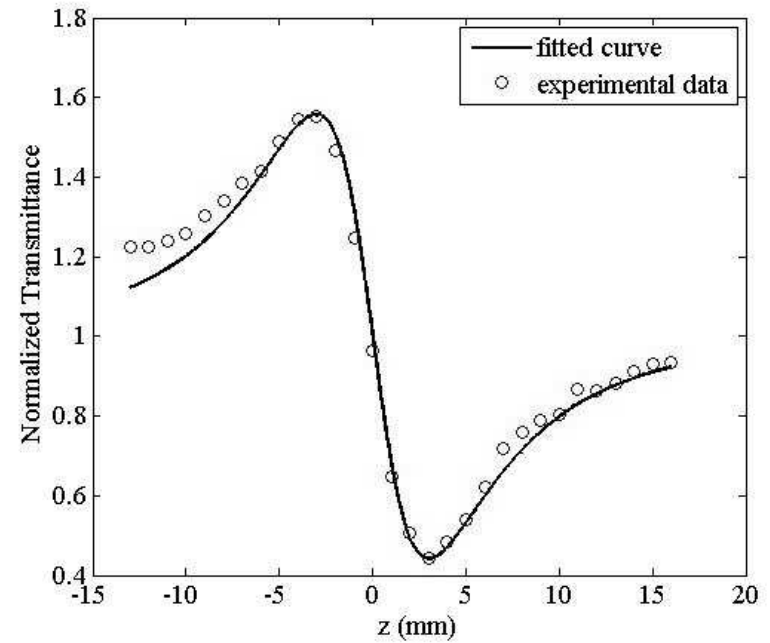

FIG. 8: Closed aperture Z-scan experimental curve for (Au-PVA) nano-fluid measured for sample with particle size of $18.7 \mathrm{~nm}$.

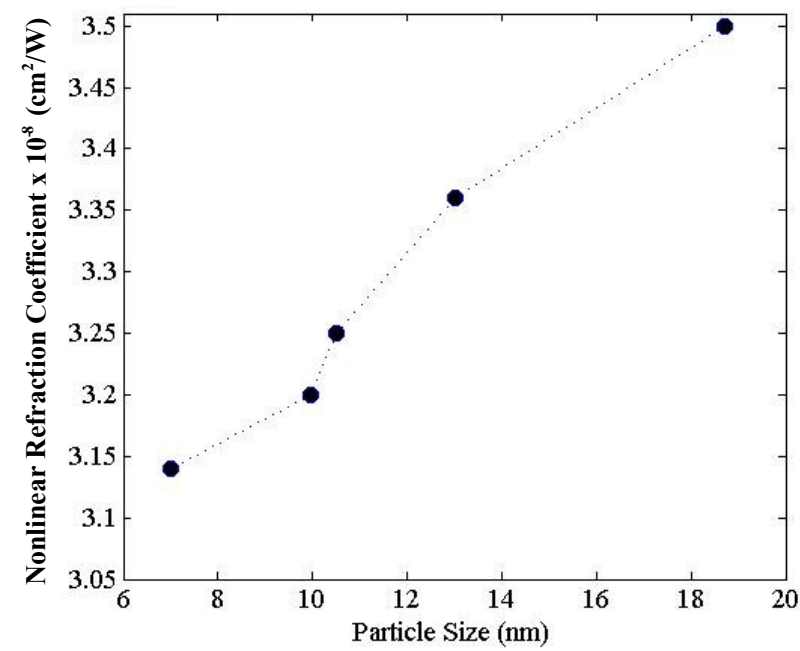

FIG. 9: Nonlinear refractive index as a function of particle size
(Table 1) in which explains the variation of the $n_{2}$ with particle size of our experimental results.

\section{CONCLUSION}

The third order nonlinearity refractive index of Au nanofluid prepared at different particles sizes has been successful measured using a single beam $\mathrm{z}$ scan method. The measurement was carried out at room temperature using a $\mathrm{CW}$ green laser beam at wavelength $532 \mathrm{~nm}$. The Au nano-fluid showed a good third order nonlinear response. The sign of the nonlinear refractive index was found to be negative and the magnitude was in the order of $10^{-8} \mathrm{~cm}^{2} / \mathrm{W}$. This nonlinear effect increases with the increasing of particles size ranging from $7.0 \mathrm{~nm}$ to $18.7 \mathrm{~nm}$

\section{ACKNOWLEDGEMENTS}

This work was supported by Fundamental research grant (01-11-08-664FR/5523664) of MOSTI, Ministry of Science, Technology and Innovation, Malaysia.
[1] Y. Shi, C. Zhang, H. Zhang, J.H. Bechtel, L.R. Dalton, B.H. Robinson, W.H. Steier, Low (Sub-1-Volt) Halfwave Voltage Polymeric Electro-optic Modulators Achieved by Controlling Chromophore Shape, Science. 288 (2000) 119-122.

[2] L.L. Beecroft, C.K. Ober, Nanocomposite Materials for Optical Applications, Chem. Mater. 9 (1997) 1302-1305.

[3] Y. Wang, Asymmetric crack problems calculated by the boundary collocation method, Acc. Chem. Res. 24 (1991) 133-139.

[4] Y. Dirix,C. Bastiaansen, W. Caseri, P. Smith, Oriented pearlnecklace arrays of metallic nanoparticles in polymers: A new route toward polarization-dependent color filters, Adv. Mater. 11 (1999) 223-226.

[5] R. Elghanian, J.J. Storhoff, R.C. Mucic, R. L. Letsinger, C. A. Mirkin, Selective Colorimetric Detection of Polynucleotides Based on the Distance-Dependent Optical Properties of Gold
Nanoparticles, Science. 277 (1997) 1078-1081.

[6] T. Taton, C. Mirkin, R. Letsinger, Scanometric DNA Array Detection with Nanoparticle Probes, Science. 289 (2000) 1757-1760.

[7] C. Y. Cao, R. Jin, C. A. Mirkin, Nanoparticles with Raman Spectroscopic Fingerprints for DNA and RNA Detection, Science. 297 (2002) 1536-1540.

[8] C. Loo, A. Lin, L. Hirsch, M. Lee, J. Barton, N. Halas, J. West, R. Drezek, Nanoshell-Enabled Photonics-Based Imaging and Therapy of Cancer, Technol. Cancer. Res. T. 3 (2004) 33-40.

[9] M. Quinten, A. Leitner, J. R. Krenn, F. R. Aussenegg, Electromagnetic energy transport via linear chains of silver nanoparticles, Opt. Lett. 23 (1998) 1331-1333.

[10] H. J Lezec, A. Degiron, E. Devaux, R. A. Linke, L. MartnMoreno, F. J. Garca-Vidal, T. W. Ebbesen, Beaming Light from a Subwavelength Aperture, Science. 297 (2002) 820- 
822.

[11] T. Thio, H. F Ghaemi, H. J Lezec, P. A. Wolff, T. W. Ebbesen, Surface-plasmon-enhanced transmission through hole arrays in Cr films, J. Opt. Soc. Am. B. 16 (1999) 1743-1748.

[12] M. L Brongersma, J. W.Hartman, H. A. Atwater, Electromagnetic energy transfer and switching in nanoparticle chain arrays below the diffraction limit, Phys. Rev. B. 62 (2000) R16356- 16359

[13] L. R. Hirsch, R. J. Stafford, J. A. Bankson, S. R. Sershen, R. E. Price, J. D. Hazle, N. J. Halas, and J. L. West, Nanoshellmediated near-infrared thermal therapy of tumors under magnetic resonance guidance, Proceeding of the Academy of Sciences (USA), PNAS. 100 (2003) 13549- 13554.

[14] M. Sheik-Bahaei, A. A. Said, T. H. Wei, D.J. Hagan, E. W. Van Stryland, Sensitive measurement of optical nonlinearities using a single beam, IEEE. J. Quant. Electron. 26 (1990) 760769.

[15] R. L. Sutherland, Handbook of Nonlinear Optics, Marcel Dekker, New York, 1996.
[16] H.B. Liao, R.F. Xiao, J.S. Fu, H. Wang, K.S. Wong, G.K.L. Wong, P. Sheng, Large third order optical nonlinearity in Au:Sio2 composite films near the percolation threshold, Appl. Phys. Lett. 70 (1997) 1-3.

[17] S.R. Marder, W.E. Torruellas, M. Blanchard-Desce, V. Ricci, G.I. Stegeman, S. Gilmour, J.L. Brédas, J. Li, G.U. Bublitz and S.G. Boxer, Large Molecular Third-Order Optical Nonlinearities in Polarized Carotenoids, Science. 276 (1997) 12331236.

[18] G. S. He, L. X. Yuan, Y. P. Cui, P. N. Prasad, Studies twophoton pumped frequency unconverted lasing properties of a new dye material, Appl. Phys. 81 (1997) 2529-2537.

[19] E. Shahriari, W.M.M. Yunus, K. Naghavi, Z.A. Talib, Effect of concentration and particle size on nonlinearity of $\mathrm{Au}$ nano-fluid prepared by $\gamma(60 \mathrm{Co})$ radiation, Opt. Commun. 283 (2010) 1929-1932.

[20] F. L. S. Anna Cuppo, A. M. Figueiredo Neto, S. L. Gomez and P. Palffy-Muhoray. JOSA B (2002) 1342-1348. 